\title{
SOFTENING-INDUCED DYNAMIC LOCALIZATION INSTABILITY: Seismic Damage in Frames
}

\author{
By Zdeněk P. Bažant, ${ }^{1}$ Fellow, ASCE, and Milan Jirásek ${ }^{2}$
}

\begin{abstract}
Aвstract: This paper analyzes dynamic localization of damage in structures with softening inelastic hinges and studies implications for the seismic response of reinforced concrete or steel frames of buildings or bridges. First, the theory of limit points and bifurcation of the symmetric equilibrium path due to localization of softening damage is reviewed. It is proven that, near the state of static bifurcation or near the static limit point, the primary (symmetric) path of dynamic response or periodic response temporarily develops Liapunov-type dynamic instability such that imperfections representing deviations from the primary path grow exponentially or linearly while damage in the frame localizes into fewer softening hinges. The implication for seismic loading is that the kinetic energy of the structure must be absorbed by fewer hinges, which means faster collapse. The dynamic localizations are demonstrated by exact analytical solutions of torsional rotation of the floor of a symmetric and symmetrically excited frame, and of horizontal shear excitation of a building column. Static bifurcations with localization are also demonstrated for a portal frame, a multibay frame, and a multibay-multistory frame. The widely used simplification of a structure as a single-degree-of-freedom oscillator becomes invalid after the static bifurcation state is passed.
\end{abstract}

\section{INTRODUCTION}

Softening damage causes localizations of damage which generally accelerate the process of collapse. If the primary response of the structure is in some sense symmetric, the localization represents a symmetry-breaking response, resulting from either a softening-induced limit point (maximum load point) or a softening-induced bifurcation of the equilibrium path.

In building frames, softening damage tends to localize into short beam segments called inelastic hinges. The localizations due to softening in inelastic hinges have been demonstrated for the static loading of reinforced concrete and steel frames (Maier 1971; Maier et al. 1973; Bažant 1976; Bažant et al. 1987; Bažant and Cedolin 1991; Hunt and Baker 1995). However, the questions of whether such localizations occur under dynamic loading and what the general character of the dynamic response of building frames with softening hinges is has escaped serious attention. These questions are addressed in this paper.

Currently, the problem is of great interest for the earthquake analysis of buildings or bridge frames. They are the principal focus of this paper although the general analysis will apply to any type of dynamic damage and multiple fracture, for example, the damage due to impact or blast. While it would be desirable to design building frames so that their load-deflection diagram would exhibit a horizontal yield plateau, in practice this usually appears impossible to achieve. This is so not only for reinforced-concrete frames, but also for steel frames.

In reinforced-concrete frames, the large deformations that occur in earthquake cause strain-softening damage in the form of distributed cracking of the material and multiple fractures. When such damage occurs only on the tensile side of the cross section, along with tensile yielding of reinforcing bars, there is no significant softening and the inelastic hinge is more precisely called the plastic hinge. But when such damage occurs

${ }^{1}$ Walter P. Murphy Prof. of Civ. Engrg. and Mat. Sci., Northwestern Univ., Evanston, IL 60208.

${ }^{2}$ Res. Assoc., Swiss Fed. Inst. of Technol. at Lausanne (EPFL), Switzerland; formerly, Asst. Prof., Czech Tech. Univ. (ČVUT), Prague, Czech Republic.

Note. Associate Editor: Raymond D. Krieg. Discussion open until May 1, 1997. To extend the closing date one month, a written request must be filed with the ASCE Manager of Journals. The manuscript for this paper was submitted for review and possible publication on March 20 , 1995. This paper is part of the Journal of Engineering Mechanics, Vol. 122, No. 12, December, 1996. CASCE, ISSN 0733-9399/96/0012$1149-1158 / \$ 4.00+\$ .50$ per page. Paper No. 10399. on the compressed side of the cross section, the inelastic hinge ceases to be plastic. Rather, development of compression splitting cracks and crushing of concrete before the yielding of steel in tension causes gradual flexural softening in which the bending moment decreases at increasing curvature [Fig. 1(a)]. Such behavior can be especially marked in prestressed beams and in reinforced-concrete beams under high axial force, as in columns of tall buildings or wide-span frames with a high horizontal thrust. The phenomenon can be particularly important for high-strength concrete, due to its extreme brittleness. Under seismic loading, the softening damage is intensified by load repetitions.

Steel frames are often thought to be free of softening because the material exhibits no strain softening. However, as shown experimentally by Maier and Zavelani (1970), the moment-rotation diagram of the plastic hinges in steel frames of large or thin-wall cross sections can develop postpeak softening as a result of plastic buckling of the flanges and webs. The development and propagation of cracks, which form in welding zones, can have the same effect.

This paper will first present a general analysis of the stability of motion of a symmetric statically indeterminate softening structure near the state of static bifurcation of the equilibrium path or near the static limit-point state. The analysis will be generally applicable to any type of dynamic loading and any kind of softening structure. Subsequently, the analysis will be applied to the seismic behavior of building frames.
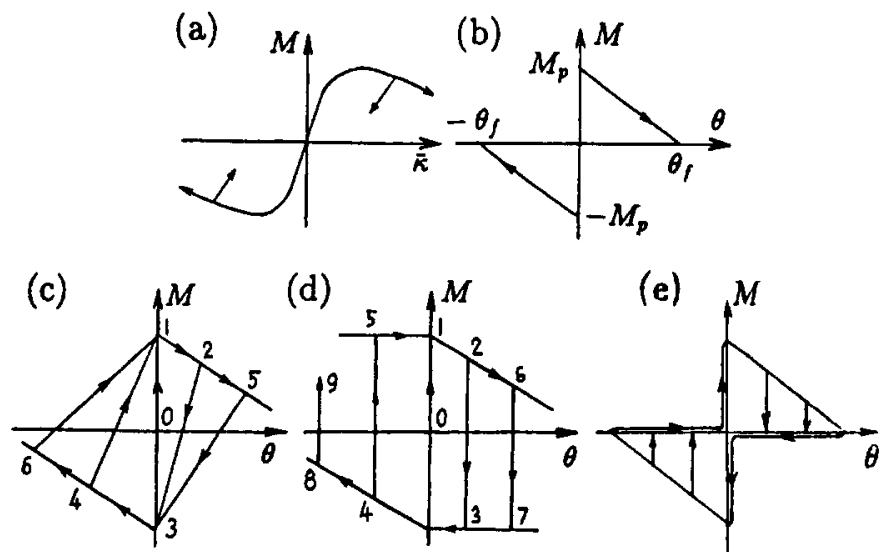

FIG. 1. (a) Diagram of Bending Moment versus Average Curvature in Softening Segment of Beam; $(b-\theta)$ Simplified MomentRotation Diagrams for Softening Hinges 
Several characteristic types of static damage localization in frames will be demonstrated and implications for the dynamic localization of damage will then be discussed.

\section{REVIEW OF EQUILIBRIUM PATH BIFURCATION AND LIMIT POINTS}

The incremental equilibrium equations of a discrete structural system may be written as $\mathbf{K} \dot{\mathbf{q}}=\mathbf{f}$ where $\mathbf{q}=$ column matrix of displacement parameters; $\bar{f}=\dot{\mu} \overline{\mathbf{f}}=$ column matrix of associated applied forces; $\mu=$ load parameter; $\overline{\mathbf{f}}=$ fixed reference loading vector; and $\mathbf{K}=n \times n$ tangential stiffness matrix of the structure (which is symmetric). The overdots denote time differentiation. As inelasticity and damage develop during the progress of loading, matrix $\mathbf{K}$ varies as a function of $\mathbf{q}$. First, we will assume this variation to be continuous but later we will consider a discontinuous change in $\mathbf{K}$ as the limit case of a rapid continuous change.

For each $\mathbf{q}$, matrix $\mathbf{K}$ also depends on the unit direction vector $\boldsymbol{v}=\dot{\mathbf{q}}\left(\dot{\mathbf{q}}^{T} \dot{\mathbf{q}}\right)^{-1 / 2}$ in the $n$-dimensional displacement rate space (superscript $T$ denotes a transpose). For simple types of constitutive laws for damage, the dependence of $\mathbf{K}$ on $\boldsymbol{\nu}$ at fixed $q$ is piecewise constant, i.e., matrix $K$ is different in various sectors of the $n$-dimensional displacement rate space, but constant within each sector (Fig. 2). Let $\mathbf{K}^{L}$ be the stiffness matrix for the loading sector $L$, for which every point of the structure undergoes loading. Adjacent to sector $L$, there are various sectors $U, U^{\prime}, U^{\prime \prime}, \ldots$, in which different combinations of loading and unloading occur in various parts of the structure. Let $U$ be the sector with unloading to which the first secondary branch actually belongs, and $\mathbf{K}^{U}$ the corresponding stiffness matrix.

The primary response path is defined as the path for which the response preserves the symmetry of the structure and all the points in the structure undergo loading. For this path, the equilibrium equations are $\mathbf{K}^{L} \dot{\mathbf{q}}^{(1)}=\dot{\mathbf{f}}$, with the direction vector $\boldsymbol{v}^{(1)}$ of $\dot{\mathbf{q}}^{(1)}$ lying in sector $L$.

We suspect that, after some state $q^{0}$ representing the first bifurcation, a second path labeled by superscript ${ }^{(2)}$ and obeying the equilibrium equation $\mathbf{K}^{U} \dot{\mathbf{q}}^{(2)}=\dot{\mathbf{f}}$ may become possible. For this path to exist, the direction vector $\boldsymbol{v}^{(2)}$ of $\dot{\mathbf{q}}^{(2)}$ must lie in sector $U$ corresponding to $\mathbf{K}^{U}$. Prior to the first bifurcation, $\nu^{(2)}$ cannot lie in the sector $U$, or else the first bifurcation could not be the first. So $v^{(2)}$ must lie either in $L$ or in some of the other sectors $U^{\prime}, U^{\prime \prime}, \ldots$, with different unloading combinations. However, none of the sectors $U^{\prime}, U^{\prime \prime}, \ldots$ is possible at the beginning of loading if we assume the stiffness matrix to vary continuously (or the stress-strain diagrams to be smooth); the reason: $\mathbf{K}^{U}$ must initially be almost the same as $\mathbf{K}^{L}$ and $\boldsymbol{v}^{(2)}$ almost the same as $\boldsymbol{\nu}^{(1)}$, since the initial difference between the tangential moduli for loading and unloading is negligible.

When $\mathbf{K}^{U}$ changes continuously, the direction of $\boldsymbol{\nu}^{(2)}$ cannot change by jumps and so $\nu^{(2)}$ must remain in $L$ for some duration of loading before it can cross into some of the sectors

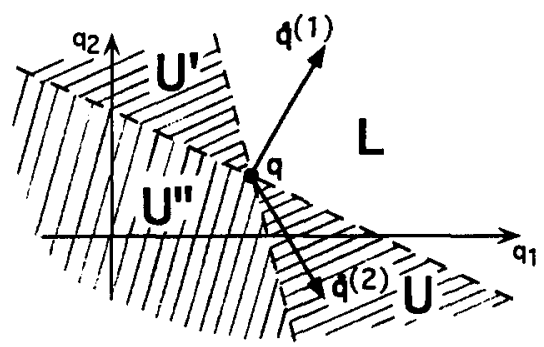

FIG. 2. Coordinate Plane of $n$-Dimensional Displacement Rate Space, with Sector of Loading Only and Sectors of Combined Loading and Unloading with unloading. Choosing proper notation, we may always assume $\nu^{(2)}$ crosses straight into $U$, without passing through some other sector with unloading such as $U^{\prime}$. Thus, at the first bifurcation, $\boldsymbol{v}^{(2)}$ becomes coincident with the boundary between sectors $L$ and $U$, and so either the secondary branch must satisfy the equation $\mathbf{K}^{L} \dot{\mathbf{q}}^{(2)}=\mathbf{f}$ simultaneously with $\mathbf{K}^{L} \dot{\mathbf{q}}^{(1)}=\mathbf{f}$ for the primary path (the first case) or, alternatively, $\mathbf{K}^{L} \dot{\mathbf{q}}^{(2)}=\mathbf{0}$ (the second case).

We immediately see that det $\mathbf{K}^{L}=0$ in the second case. In the first case, on subtracting both equations we get

$$
\mathbf{K}^{L} \mathbf{w}=0 ; \quad \dot{\mathbf{w}}=\dot{\mathbf{q}}^{(2)}-\dot{\mathbf{q}}^{(1)}
$$

(This condition is the same as the condition of bifurcation of the equilibrium path of an elastic structure.) Eq. (1) represents a system of homogeneous linear algebraic equations for the eigenvector $\dot{w}$. Obviously, for the secondary branch to exist, i.e., $\dot{w} \neq 0$, it is necessary that

$$
\operatorname{det} \mathbf{K}^{L}=0
$$

which is the same condition as in the second case. So, in both cases, $\mathbf{K}^{L}$ must be singular, i.e., the first (lowest) eigenvalue of matrix $\mathbf{K}^{L}, \lambda_{1}^{K}=0$. After passing the first bifurcation point, $\lambda_{1}^{K}$ is normally negative.

If the constitutive law for inelastic behavior is not smooth, which, for example, happens for a bilinear stress-strain diagram, the tangential stiffness matrix $K$ as a function of $q$ varies by jumps. In that case, the change of the first eigenvalue $\lambda_{1}^{K}$ from positive to nonpositive is in general discontinuous, and the matrix $\mathbf{K}$ for the initial bifurcating secondary branch is $\mathbf{K}^{U}$, which corresponds to a combination of loading and unloading in various parts of the structure rather than to loading only. This case may be conveniently viewed as the limit case of a continuous change of $\mathbf{K}$ as a function of $\mathbf{q}$ in which the first eigenvalue crosses zero very rapidly. From this viewpoint, the negativeness of the first eigenvalue is equivalent to the foregoing case of continuous stiffness variation for which the bifurcation point has already been passed. Thus, if the first eigenvalue becomes negative, a bifurcating secondary branch must exist, although in general it corresponds to $\mathbf{K}^{U}$, which is different from $\mathbf{K}^{L}$.

The previous analysis shows when a bifurcation of the equilibrium path into a secondary branch is possible, but does not show which branch is actually followed by the structure. However, analysis of the second energy variations and use of the second law of thermodynamics prove that the structure must follow the secondary branch. For more detail, see Bažant and Cedolin [(1991), sections 10.2 and 10.4]; also see de Borst (1989), Stoltz (1989), Borrè and Maier (1989), Runesson et al. (1989), and Leroy and Ortiz (1989).

A caveat should be mentioned: since the equilibrium path bifurcation can be detected only on the basis of the tangential stiffness matrix, the nonpositiveness of the first eigenvalue (or loss of positive definiteness of $\mathbf{K}^{L}$ ) can remain unnoticed by finite-element programs that do not work with the tangential stiffness matrix. After the bifurcation state, the iteration process in such programs can converge to the primary branch, which is normally the unstable branch. Thus the more important secondary branch, which is normally the stable path, is missed. This of course happens not only for elastic, but also for inelastic structures. The reason, briefly, is that the initial approximate solution in a loading step exhibits the same type of symmetry as the primary branch, while the stable secondary branch breaks the symmetry - it departs from the bifurcation point at an inclined direction that contains a nonsymmetric component. In numerical solution, round-off errors might be insufficient to provide an initial nonsymmetric imperfection which could trigger the break of symmetry.

To detect a secondary branch, the finite-element code must 
calculate the tangential stiffness matrix and check the sign of its lowest eigenvalue. If the sign is not positive, the code must automatically seek a displacement increment for which a part of the structure goes into unloading.

\section{DYNAMIC INSTABILITY NEAR STATIC BIFURCATION OR LIMIT POINT}

Consider now what happens near the static bifurcation state under dynamic loading. A bifurcation in time, similar to the one we demonstrated for static response, is impossible because it would imply a sudden change of some components of velocity. This would mean that the associated components of acceleration vector $\ddot{q}$ would be infinite, which is impossible provided that the applied forces remain finite. For a given excitation and given initial conditions, the dynamic solution is unique and cannot exhibit bifurcations in time.

Of course, bifurcations can be observed in dynamics if one studies the dependence of a periodic solution on the variation of a certain parameter, e.g., the period of excitation (Guckenheimer and Holmes 1983; Wiggins 1990). However, this is a different type of problem not considered here. We focus only on the response of a system for fixed parameter values.

Let $\mathbf{q}^{(1)}(t)$ (where $t=$ time) be the solution vector for given initial conditions $\mathbf{q}^{\text {in }}$ and given load vector history $\mathbf{f}(t)$, called the primary response. Let us now examine what happens when these initial conditions are slightly perturbed, i.e., changed to $\mathbf{q}^{i n}+\mathbf{w}^{i n}$. This leads to a different solution vector $\mathbf{q}^{(2)}(t)$, called the perturbed (or secondary) response. As long as the tangential stiffness $\mathbf{K}$ is positive definite (i.e., all its eigenvalues are positive), the solution vectors $\mathbf{q}^{(1)}(t)$ and $\mathbf{q}^{(2)}(t)$ must remain very close. Thus, their difference in displacement, $\mathbf{w}=\mathbf{q}^{(2)}-$ $\mathbf{q}^{(1)}$, remains very small until time $t_{0}$ at which the state of static bifurcation (or limit point) is approached. So, $\mathbf{w}_{0}=\mathbf{w}\left(t_{0}\right)$ must be very small.

Denote $\mathbf{q}_{0}=$ displacement at the state of static bifurcation (or limit point). In the vicinity of this state, the vector of static forces due to the deformation of the inelastic structure can be tangentially approximated as $\mathbf{f}_{\text {def }}(t)=\mathbf{K}\left[\mathbf{q}(t)-\mathbf{q}_{0}\right]+\mathbf{f}_{0}$, where $\mathbf{K}$ is the tangential stiffness matrix of the structure for loading only at the state of static bifurcation (or limit point) and $\mathbf{f}_{0}$ is the static force vector in this state. On adding the force vectors due to inertia and damping, we obtain the following equations of motion near the state of static bifurcation (or limit point):

$$
\begin{aligned}
& \mathbf{M} \ddot{q}^{(1)}+\mathbf{D} \dot{\mathbf{q}}^{(1)}+\mathbf{K}\left[\mathbf{q}^{(1)}-\mathbf{q}_{0}\right]+\mathbf{f}_{0}=\mathbf{f}(t) \\
& \mathbf{M} \ddot{\mathbf{q}}^{(2)}+\mathbf{D} \dot{\mathbf{q}}^{(2)}+\mathbf{K}\left[\mathbf{q}^{(2)}-\mathbf{q}_{0}\right]+\mathbf{f}_{0}=\mathbf{f}(t)
\end{aligned}
$$

where superscripts ${ }^{(1)}$ and ${ }^{(2)}$ label the primary response and the perturbed (secondary) response; and $\mathbf{M}$ and $\mathbf{D}=$ mass and damping matrices. Because the responses 1 and 2 remain very close to each other up to and shortly after the static bifurcation (or limit) point, the same tangential stiffness matrix $\mathbf{K}$ and the same vector $\mathbf{f}_{0}$ is applicable to both. According to the preceding analysis of static bifurcation, $\mathbf{K}=\mathbf{K}^{L}$.

Subtracting (3) from (4), we obtain for the difference $\mathbf{w}$ in the displacement vectors the following equation of motion:

$$
\mathbf{M} \ddot{\mathbf{w}}+\mathbf{D} \dot{\mathbf{w}}+\mathbf{K}^{L} \mathbf{w}=\mathbf{0}
$$

which is valid only for a short enough time interval after the static bifurcation state. The special case of this equation for static response $(\mathbf{M}=\mathbf{D}=\mathbf{0})$ is (1).

Consider now dynamic response $(\mathbf{M} \neq \mathbf{0})$ with no damping $(D=0)$. In the vicinity of the static bifurcation state, $K^{L}$ can be considered constant and the displacement difference can be sought in the form $\mathbf{w}=\mathbf{a} e^{i \omega t}$ in which $i=$ imaginary unit; $\omega$ $=$ constant; and $\mathbf{a}=$ amplitude vector (column matrix). Substituting this into (5) we obtain

$$
\left(\mathbf{K}^{L}-\omega^{2} \mathbf{M}\right) \mathbf{a}=\mathbf{0}
$$

This is a homogeneous linear matrix equation representing a generalized eigenvalue problem. Because matrix $\mathbf{M}$ is always positive definite, each eigenvalue $\lambda=\omega^{2}$ of this matrix equation has the same sign as the corresponding eigenvalue of matrix $\mathbf{K}$ and is zero if the eigenvalue of $\mathbf{K}$ is zero.

So the first eigenvalue $\lambda_{1}$ of matrix equation (6) is zero at the first static bifurcation state if matrix $\mathbf{K}^{2}$ evolves continuously, and is nonpositive if $\mathbf{K}^{L}$ evolves discontinuously (which is, for example, the case for softening materials with a bilinear constitutive law). Because, after the state of static bifurcation, $\omega^{2}=\lambda_{1} \leq 0$, we may set $\omega= \pm i \lambda$ in which $\lambda=\sqrt{-\lambda_{1}}=$ real. So we conclude that the displacement difference for a short enough time after the static bifurcation state must have the following form:

$$
\begin{gathered}
\mathbf{w}=\mathbf{q}^{(2)}-\mathbf{q}^{(1)}=\dot{w}^{0} t+\mathbf{w}^{0}+\mathbf{w}^{H}(t) \text { if } \quad \lambda_{1}=0 \\
\mathbf{w}=\dot{\mathbf{w}}^{0} \frac{\sinh \lambda t}{\lambda}+\mathbf{w}^{0} \cosh \lambda t+\mathbf{w}^{H}(t) \text { if } \quad \lambda_{1}<0
\end{gathered}
$$

in which $\mathbf{w}^{0}$ and $\dot{\mathbf{w}}^{0}=$ small differences in displacements and velocities between the primary response and the initially slightly perturbed response; and $\mathrm{w}^{H}(t)=$ terms corresponding to higher eigenvalues.

The foregoing analysis assumes $\lambda_{1}$ not to be a double root. If it is, terms with $t^{2}$ or $t \sinh \lambda t$ and $t \cosh \lambda t$ need to be added to (7) or (8), but the conclusions remain the same.

Imperfections in structures are never zero. No matter how small $\dot{w}^{0}$ or $w^{0}$ at the state of bifurcation, the difference $w$ according to (7) or (8) will subsequently grow and deviate from the primary response. This indicates a dynamic instability of the primary solution, which is normally of limited duration (temporary). Of course, the stiffness matrix might later become positive definite again, in which case dynamic stability will be restored. But even in that case, the initial imperfections will have been magnified by the time the lowest eigenvalue of $\mathbf{K}$ might change its sign again.

So we conclude that the primary (unperturbed) solution, corresponding to the symmetric response, becomes temporarily unstable when the state of bifurcation of the equilibrium path is reached. The instability is the dynamic instability in the sense of Liapunov [e.g., Bažant and Cedolin (1991), Chapter 3]. This means that in reality the primary or symmetric solution beyond the static bifurcation state cannot occur. Before the state of static bifurcation is reached, any small perturbations of the symmetric response remain small. But after that state, they grow approximately exponentially (because $\sinh \lambda t$ and $\cosh \lambda t$ are linear combinations of $e^{\lambda t}$ and $\left.e^{-\lambda t}\right)$.

A system is defined as dynamically stable (in the sense of Liapunov) if, for any arbitrarily small positive number $\varepsilon$, there exists a positive number $\delta$ such that any deviation $\mathbf{w}^{0}$ and $\dot{w}^{0}$, at time $t_{0}$, having a magnitude not larger than $\delta$ leads to a solution $\mathbf{w}(t)$ and $\mathbf{w}(t)$ whose magnitude remains smaller than $\varepsilon$ for all times $t \geq t_{0}$ [see, e.g., Bažant and Cedolin (1991), Section 3.5]. Otherwise, the system is dynamically unstable.

If an exponential term with a positive exponent (or a linear term) is present in the solution, as in (8), the system is obviously unstable, according to this definition. It must be admitted that in practice the exponential (or linear) growth of response deviation cannot be considered to go on up to infinite time, because the lowest eigenvalue of $\mathbf{K}$ will change its sign or because the deviation from the state of static bifurcation ceases to be small, making our linearized solution invalid. One may emphasize this point by calling the exponential growth of response deviation as a "temporary" (or "time-limited") dynamic instability. But the adjective temporary is not really necessary because it is obvious. Similar limitations occur in most practical situations in which the term "dynamic instability" is 
in standard use. For example, the exponential growth of vibration amplitudes of a dynamically unstable elastic column ceases when the range of geometrically nonlinear large deflections is entered; the exponential growth of the vibration amplitudes of a dynamically unstable aircraft wing failing by flutter (or a dynamically unstable shaft rotating at a critical speed) ceases when the amplitudes become so large that the linearized small deflection theory becomes invalid, etc. [see e.g., Bažant and Cedolin (1991), Chapter 3].

For the secondary solution, some softening parts of the structure undergo unloading. The softening parts that are unloading and those that are not can be determined by static bifurcation analysis, from the eigenvector of displacement velocity, $\dot{w}$, corresponding to the first eigenvalue of the tangential stiffness matrix for loading only.

It may be objected that bifurcation points are not generic. Real structures, strictly speaking, do not exhibit bifurcations because they are never perfectly symmetric, due to inevitable small imperfections. However, this does not mean that the bifurcation points would be unimportant. The analysis of bifurcation gives information on the entire behavior near the bifurcation. For example, the Euler bifurcation is actually never experienced by real elastic columns, because of imperfections, yet column buckling and its imperfection sensitivity could not be understood without knowing the critical load and understanding the Euler bifurcation.

The foregoing solution applies to the motion near the static bifurcation state. The solution of motion near the static limit point (maximum load) is similar but its character is deduced more simply. It suffices to say that, because of the existence of a negative eigenvalue of $\mathbf{K}$ after the static limit point, the general solution of the equations of motion must contain growing exponential terms. Therefore, small deviations from the primary solution for the symmetric structure can grow rapidly. This represents again a (temporary) dynamic instability (of Liapunov type). It is not necessary for the unperturbed dynamic solution to exhibit all the symmetries of the primary static solution (this will become clear when we analyze the dynamic shearing of a column).

Consider now the effect of damping. First, let us look at a system with a single degree of freedom. The equation $M \ddot{q}+$ $D \dot{q}+K q=0$-in which constants $M, D$, and $K$ represent the mass, damping, and stiffness - has the solution $q(t)=e^{\lambda s}$ with $\lambda=\left(-D \pm \sqrt{D^{2}-4 M K}\right) / 2 M$. The case of negative damping, $D<0$, may be excluded. Instability occurs if $\lambda$ has a positive real part, i.e., if $K<0$, regardless of the value of $D$. So the damping has no influence on the question of stability.

A similar analysis for a damped multidegree-of-freedom system shows that the response deviation $\mathbf{w}(t)$ will start to increase exponentially regardless of $D$ as soon as a negative eigenvalue of $\mathbf{K}$ appears, provided that the type of damping is such that the degrees of freedom can be decoupled by introducing as generalized coordinates the eigenvectors of $\mathbf{K}$ with respect to $\mathbf{M}$. This is true, for example, for a damping matrix $\mathbf{D}$ proportional to the mass matrix $\mathbf{M}$. This is probably also true for the general damping of earthquake-type excitation because the only cases for which damping is known to affect stability (i.e., stabilize or destabilize a dynamic system) are the parametric resonance and gyroscopic loads [Bažant and Cedolin (1991), p. 166 and 173].

\section{SEISMIC INSTABILITIES OF FRAMES WITH SOFTENING HINGES}

One important application of the preceding general theory is the instability of symmetric response of frames with softening hinges subjected to earthquake. In slender frames, which respond primarily by bending with shear, the inelastic defor- mation concentrates into short beam segments of high average curvature $\bar{\kappa}$. The diagram of bending moment $M$ versus $\bar{\kappa}$ typically exhibits postpeak softening, as shown in Fig. 1(a). As a simplifying idealization, the inelastic response can be idealized by lumping it into a single cross section regarded as an inelastic hinge, while the rest of the beam is assumed to respond elastically. The relative rotation $\theta$ in the inelastic hinge represents the rotation difference between the ends of the hinging segment caused by the inelastic part of the curvature.

To simplify calculations, the moment-rotation diagram may be idealized as linear [Fig. 1(b)]. It is characterized by peak moment $M_{p}$ and fracture rotation $\theta_{f}$ at which $M$ is reduced to zero. With regard to unloading and reloading, the behavior may be idealized according to one of the diagrams in Figs. $1(c-e)$.

To illustrate the preceding general theory, we will now consider four basic types of static bifurcation in building frames and the corresponding (temporary) dynamic instabilities.

\section{Localization due to Rotation of Bullding Floor}

Consider a rectangular slab (building floor) supported symmetrically by two rows of columns and dynamically excited by foundation movement $q(t)$ in the direction of the column rows [Fig. 3(a)]. The problem is idealized [Fig. 3(b)] as a rigid block sliding on frictionless supports and excited through a symmetric pair of "springs" which follow the bilinear forcedisplacement diagram in Fig. 3(c); $C_{e}=$ spring constant (elastic stiffness), $C_{s}=$ postpeak softening stiffness of spring $\left(C_{s}<0\right)$, $F_{p}=$ peak force, $u_{p}=$ displacement at peak, and $u_{f}=$ fracture displacement at which $F$ is reduced to zero. The springs are attached to another rigid block whose displacement history $q(t)$ is prescribed to simulate the seismic excitation.

Let us first look at the corresponding static problem of a floor symmetrically loaded by a displacement $u$ imposed at its center. Any static solution must satisfy the conditions of equilibrium

$$
F=F_{1}+F_{2} ; \quad L F_{1}=L F_{2}
$$

where $F_{1}$ and $F_{2}=$ forces transmitted by springs 1 and 2 , respectively; and $L=$ half of the distance between the springs. For a piecewise linear force-displacement diagram, the force in spring $i$ can in general be written as

$$
F_{i}=F_{i}^{0}+C_{i} u_{i} \quad(i=1,2)
$$
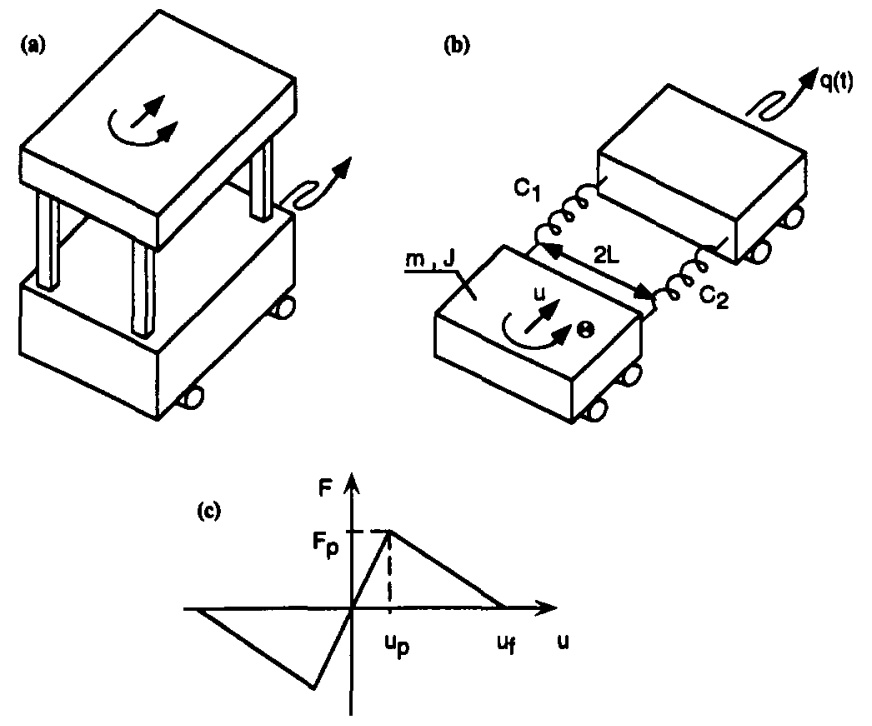

FIG. 3. (a) Dynamicaliy Excited Bullding Floor; (b) Simplified Model; (c) Bilinear Force-Extension Diagram for Springs Modelling the Columns 
where $F_{i}^{0}$ and $C_{i}=$ constants whose values depend on the currently active branch of the force-displacement diagram. During virgin loading and elastic unloading, $F_{i}^{0}=0$ and $C_{i}=C_{e}$, while during softening, $F_{i}^{0}=F_{p} u_{f} /\left(u_{f}-u_{p}\right)$ and $C_{i}=C_{s}$. Substituting (10) into (9) and transforming to variables $u=\left(u_{1}+u_{2}\right) / 2$ and $v=\left(u_{2}-u_{1}\right) / 2$, we obtain

$$
\begin{gathered}
\left(C_{1}+C_{2}\right) u+\left(C_{2}-C_{1}\right) v=F-F_{1}^{0}-F_{2}^{0} \\
\left(C_{2}-C_{1}\right) u+\left(C_{1}+C_{2}\right) v=F_{1}^{0}-F_{2}^{0}
\end{gathered}
$$

During the initial elastic stage, $C_{1}=C_{2}=C_{e}, F_{1}^{0}=F_{2}^{0}=0$, and thus $u=F / 2 C_{e}, v=0$. Note that $v=\theta L$ where $\theta$ is the floor rotation. Therefore, the initial response is symmetric and the peak force $F_{p}$ is reached simultaneously by both springs at $u=u_{p}$. After that, either the response will remain symmetric with both springs softening, or the symmetry will be broken and damage will localize into one spring while the other unloads elastically.

Because the state at peak is an equilibrium state, (11) and (12) can be rewritten in an incremental form as

$$
\begin{gathered}
\left(C_{1}+C_{2}\right) \Delta u+\left(C_{2}-C_{1}\right) \Delta v=\Delta F \\
\left(C_{2}-C_{1}\right) \Delta u+\left(C_{1}+C_{2}\right) \Delta v=0
\end{gathered}
$$

In the symmetric case, $C_{1}=C_{2}=C_{s}$, (13) and (14) yield $\Delta u$ $=\Delta F / 2 C_{s}$, and $\Delta v=0$. In the nonsymmetric case with, e.g., spring 1 unloading and spring 2 softening, we have $C_{1}=C_{e}$ $>0, C_{2}=C_{s}<0$, and (13) and (14) yield

$$
\begin{aligned}
& \Delta u=\frac{C_{1}+C_{2}}{4 C_{1} C_{2}} \Delta F=\frac{C_{e}+C_{s}}{4 C_{e} C_{s}} \Delta F \\
& \Delta v=\frac{C_{1}-C_{2}}{4 C_{1} C_{2}} \Delta F=\frac{C_{e}-C_{s}}{4 C_{e} C_{s}} \Delta F
\end{aligned}
$$

As we have shown, the equilibrium response path of the floor bifurcates at the peak load state, that is, at the start of softening. Similarly, it can be shown that there is a bifurcation at every point (e.g. point 4 in Fig. 4) along the symmetric response path (the straight line connecting the peak to point $f$ ), such that one spring starts to unload while the other spring continues softening. But what matters is the first bifurcation, which occurs at the peak. Now, which of the two postbifurcation paths will actually occur? It has been generally proven in Bažant and Cedolin [(1991), chapters 10 and 13] and Bažant (1988), on the basis of thermodynamic considerations, that the actual path is that which descends more to the left, i.e., that for which the compliance $\Delta u / \Delta F$ is larger. So, the nonsymmetric path will occur if and only if

$$
\frac{C_{e}+C_{s}}{4 C_{e} C_{s}}>\frac{1}{2 C_{s}}
$$

It can be easily verified that this is equivalent to $C_{s}<C_{e}$, which is for $C_{e}>0$ and $C_{s}<0$ always satisfied. This means that the symmetric solution is not stable and can never occur in reality. Damage will localize into one of the two springs depending on random imperfections. This process is stable under displacement control assuming that $C_{e}+C_{s}>0$, i.e., $C_{s}>-C_{e}$.

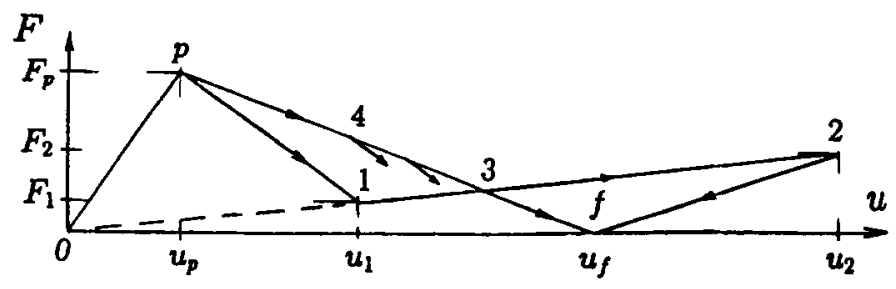

FIG. 4. Primary and Secondary Equllibrlum Response of Building Floor, Column, or Portal Frame
For $C_{s} \leq-C_{e}$, the postpeak branch corresponding to the unsymmetric solution has a positive (or infinite) slope, and the diagram exhibits snapback.

Let us now turn attention to the dynamic problem. The equations of motion for the floor as a system with two degrees of freedom can be written as

$$
\begin{gathered}
m \ddot{u}(t)+F_{1}(t)+F_{2}(t)=0 \\
J \ddot{\theta}(t)-L F_{1}(t)+L F_{2}(t)=0
\end{gathered}
$$

where $m=$ mass of the floor; $J=$ mass moment of inertia; $u$ $=\left(u_{1}+u_{2}\right) / 2=$ displacement of the floor center; and $\theta=\left(u_{2}\right.$ $\left.-u_{1}\right) / 2 L=$ rotation of the floor (positive if counterclockwise).

Eq. (10) describing the constitutive behavior must be generalized to

$$
F_{i}=F_{i}^{0}+C_{i}\left(u_{i}-q\right)
$$

as the spring extension is now the difference between the displacements $u_{i}$ and $q$ (in the static case we did not need to introduce $q$ because the base did not move). On substituting (20) into (18) and (19) and transforming to variables $u=\left(u_{1}\right.$ $\left.+u_{2}\right) / 2$ and $v=\left(u_{2}-u_{1}\right) / 2=L \theta$, we obtain

$$
\begin{aligned}
& m \ddot{u}(t)+\left(C_{1}+C_{2}\right) u(t)+\left(C_{2}-C_{1}\right) v(t) \\
& \quad=\left(C_{1}+C_{2}\right) q(t)-F_{1}^{0}-F_{2}^{0} \\
& M \ddot{v}(t)+\left(C_{2}-C_{1}\right) u(t)+\left(C_{1}+C_{2}\right) v(t) \\
& \quad=\left(C_{2}-C_{1}\right) q(t)+F_{1}^{0}-F_{2}^{0}
\end{aligned}
$$

where $M=J / L^{2}$.

During the initial elastic stage, $C_{1}=C_{2}=C_{e}, F_{1}^{0}=F_{2}^{0}=0$, and the solution of (22) is

$$
v(t)=\theta_{0} L \cos \omega_{2} t+\frac{\dot{\theta}_{0} L}{\omega_{2}} \sin \omega_{2} t
$$

where $\omega_{2}=\sqrt{2 C_{e} / M}$; and $\theta_{0}$ and $\dot{\theta}_{0}=$ initial floor rotation and its initial rate, respectively. If the initial conditions are symmetric, i.e., $\theta_{0}=\dot{\theta}_{0}=0$, we have $v(t)=0$ and the response remains symmetric. But even if a random disturbance causes a slight deviation from perfect symmetry, the deviation amplitude does not grow and the deviation only oscillates about the perfectly symmetric solution.

After both springs reach the peak force at time $t_{p}$ and some positive velocity $\dot{u}_{p}$, they start softening simultaneously. We now have $C_{1}=C_{2}=C_{s}<0$, and the solution of $(22)$ is

$$
v(t)=v_{p} \cosh \alpha_{2} t+\frac{\dot{y}_{p}}{\alpha_{2}} \sinh \alpha_{2} t
$$

where $\alpha_{2}=\sqrt{-2 C_{2} / M}$; and $v_{p}, \dot{v}_{p}=$ floor rotation and floor rotation rate at $t=t_{p}$ (assumed to be very small perturbations). If $v_{p}=\dot{v}_{p}=0$, we still have a symmetric solution with $v(t)=$ 0 . However, any small deviation from perfect symmetry now grows approximately exponentially. Thus the symmetric response of the floor is for a limited time dynamically unstable. Even if the building is almost perfectly symmetric and the earthquake strikes exactly in the direction of the column rows, the building floor must exhibit torsional rotations during the earthquake.

Seismic torsional rotations of building floors have been studied, for example, by Delallera and Chopra $(1994,1995)$. The necessity of some torsional rotations has already been recognized in the existing seismic building code by requiring that at least $5 \%$ of the displacement must be considered to be due to rotation. However, such a simple specification might not be sufficiently realistic. The magnitude of rotation that develops depends on the magnitude of imperfections; geom- 
etry, stiffness, and mass characteristics of the building frame; excitation history; and earthquake duration.

\section{Localization in Shear Loading of Building Column}

A column of a building frame responds to an earthquake principally by shear. The column may be idealized as shown in Fig. 5(a), in which both ends slide horizontally (horizontal motion of the base is prescribed) but are fixed against rotation.

First consider the static response, for which we assume the bottom of the column to be fixed and the horizontal displacement $u$ on top to be prescribed. The column fails by softening hinges which form at the ends. The peak value of the horizontal load $F$ is $F_{p}=2 M_{p} / L$ and the corresponding displacement is $u_{p}=M_{p} L^{2} / 6 E I$.

One possible postpeak response is symmetric [Fig. 5(b)], with equal magnitudes of bending moments and hinge rotations at the ends. The load-deflection diagram is linear and is given by line $p f$ in Fig. 4 , in which $u_{f}=\theta_{f} L$ where $L$ is the height of the column.

There exists another possible response, representing the bifurcating secondary branch. It is nonsymmetric, such that the bottom hinge first rotates while the top cross section (a potential hinge) unloads elastically from the maximum moment state [Fig. 5(c)]. The path is represented by line $p 1$ in Fig. 4. The terminal point 1 of this path, corresponding to $M=0$ and $\theta=$ $\theta_{f}$ at the bottom end, is found by elastic deformation analysis to be $u_{1}=2 L \theta_{f} / 3$ and $F_{1}=2 E I \theta_{f} / L^{2}$.

The requirement that the moment on top corresponding to point 1 must not exceed $M_{p}$ yields the condition

$$
\theta_{f} \leq \frac{M_{p} L}{2 E I}
$$

Otherwise, the nonsymmetric response does not exist and point $p$ represents a limit point, at which $\lambda$ becomes negative.

After point 1 , the column loads elastically along path 12 and at point 2 the moment on top reaches $M_{p}$. At point $2, u_{2}$ $=M_{p} L^{2} / 3 E I$ and $F_{2}=M_{p} / L$. The areas under both load-deflection diagrams must be equal to the energy dissipated in the softening hinges, which is $W_{f}=M_{p} \theta_{f}$. For this reason area $0 p f 0=$ area $0 p 12 f 0$. So, point 2 must lie above the path for the symmetric response, such that area $p 13 p=$ area $f 32 f$.

Fig. 6 shows a simple dynamic model of the column. The inertial properties of the column are represented by three lumped masses with no rotational inertia so that the rotational degree of freedom $\vartheta_{2}$ can be eliminated from the equations of motion by static condensation. The remaining degrees of freedom $u_{1}$ and $u_{2}$ represent the displacement of the top and center, respectively, while the displacement history $u_{3}(t)$ at the base

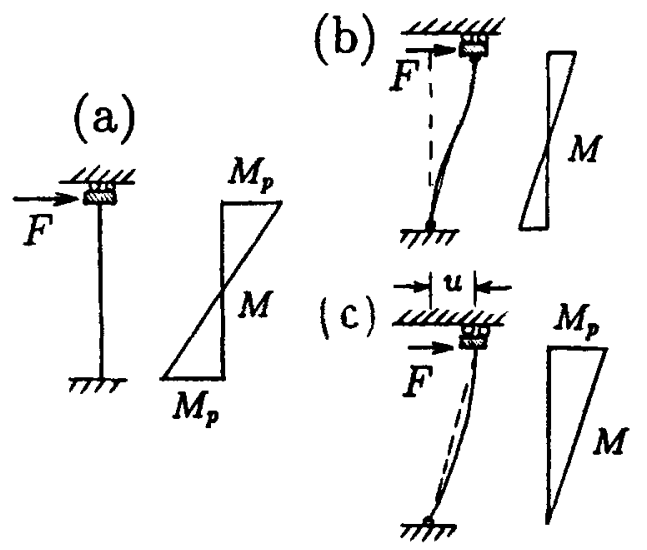

FIG. 5. Column Loaded by Horizontal Load, Its Softening Hinges, and Symmetric and Nonsymmetric Equilibrium Deflectlons

1154 / JOURNAL OF ENGINEERING MECHANICS / DECEMBER 1996

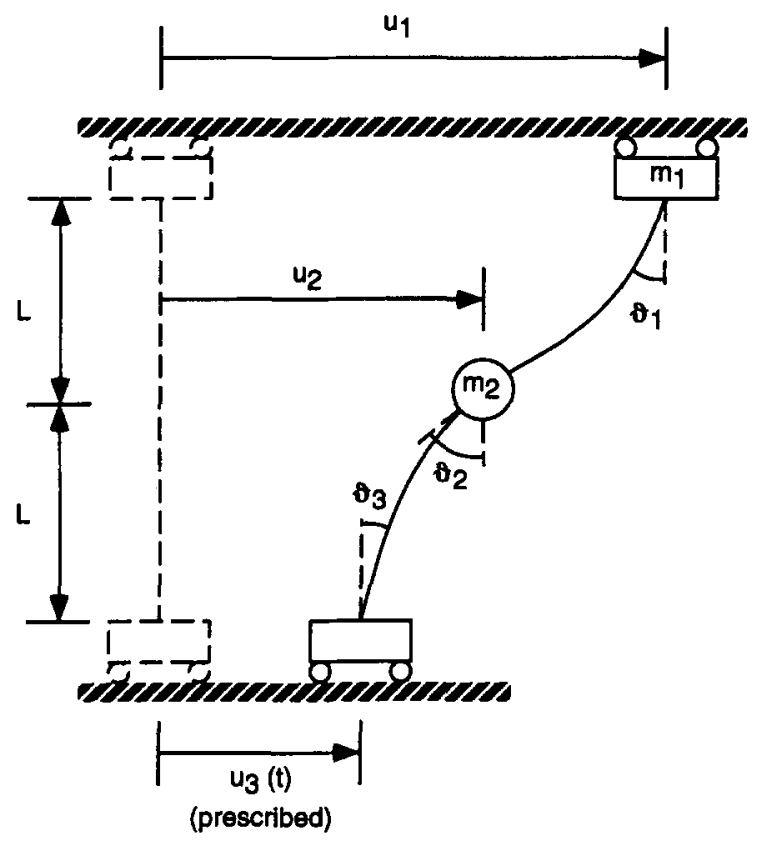

FIG. 6. Dynamic Model of Horizontally Vibrating Column

is assumed to be prescribed. The resulting equations of motion read

$$
\begin{array}{r}
m_{1} \ddot{u}_{1}+k\left(30 u_{1}-48 u_{2}+18 u_{3}-9 L \theta_{1}+3 L \theta_{3}\right)=0 \\
m_{2} \ddot{u}_{2}+k\left(-48 u_{1}+96 u_{2}-48 u_{3}+12 L \theta_{1}-12 L \theta_{3}\right)=0
\end{array}
$$

where $k=2 E I / L^{3}$. The inelastic rotations in softening hinges $\theta_{1}$ and $\theta_{3}$ must be determined by substituting the end moments

$$
\begin{gathered}
M_{1}=\frac{k L}{2}\left(18 u_{1}-24 u_{2}+6 u_{3}-7 L \theta_{1}+L \theta_{3}\right) \\
M_{3}=\frac{k L}{2}\left(-6 u_{1}+24 u_{2}-18 u_{3}+L \theta_{1}-7 L \theta_{3}\right)
\end{gathered}
$$

into the moment-rotation law. Depending on which branch of the moment-rotation diagram is currently active, three basic situations can be distinguished:

1. Both hinges "locked" (elastic loading/unloading). During virgin loading or elastic unloading, the hinge rotations remain constant. After substituting their values and the given time evolution of $u_{3}(t)$ into (26) and (27), we obtain a system of two differential equations for unknown functions $u_{1}(t), u_{2}(t)$.

2. One hinge softening, the other one locked. If one of the hinges, e.g., hinge 1 , follows the descending branch of the moment-rotation diagram, the relation between $M_{1}$ and $\theta_{1}$ is given by the softening law $M_{1}=M_{p}-\left(M_{p}\right)$ $\left.\theta_{f}\right) \theta_{1}$. This law combined with (28) can be used to express $\theta_{1}$ in terms of the displacements $u_{1}, u_{2}, u_{3}$, and the known (constant) rotation $\theta_{3}$. Again, substituting into (26) and (27), we obtain a system of two differential equations for $u_{1}(t)$ and $u_{2}(t)$.

3. Both hinges softening. As in the preceding case, we combine the softening laws $M_{1}=M_{p}-\left(M_{p} / \theta_{f}\right) \theta_{1}$ and $M_{3}=$ $M_{p}-\left(M_{p} / \theta_{f}\right) \theta_{3}$ with (28) and (29) to express $\theta_{1}$ and $\theta_{3}$ in terms of $u_{1}, u_{2}, u_{3}$, and substitute these expressions into (26) and (27).

In each of the foregoing situations, we obtain a system of two linear, second-order differential equations with constant coefficients, which can be easily solved in a closed form. For each time interval in which the status (loading or unloading) 
(a)

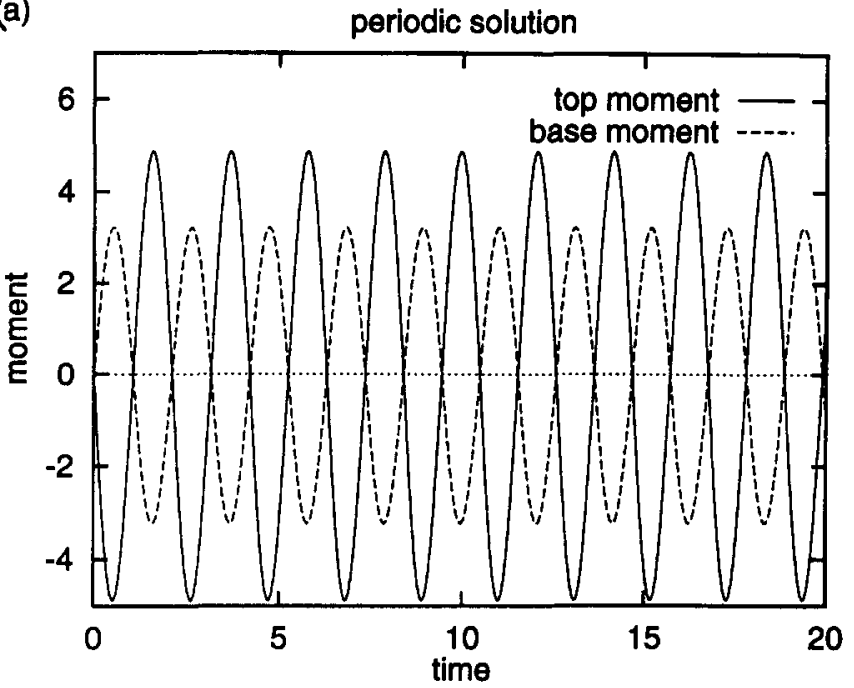

(b)

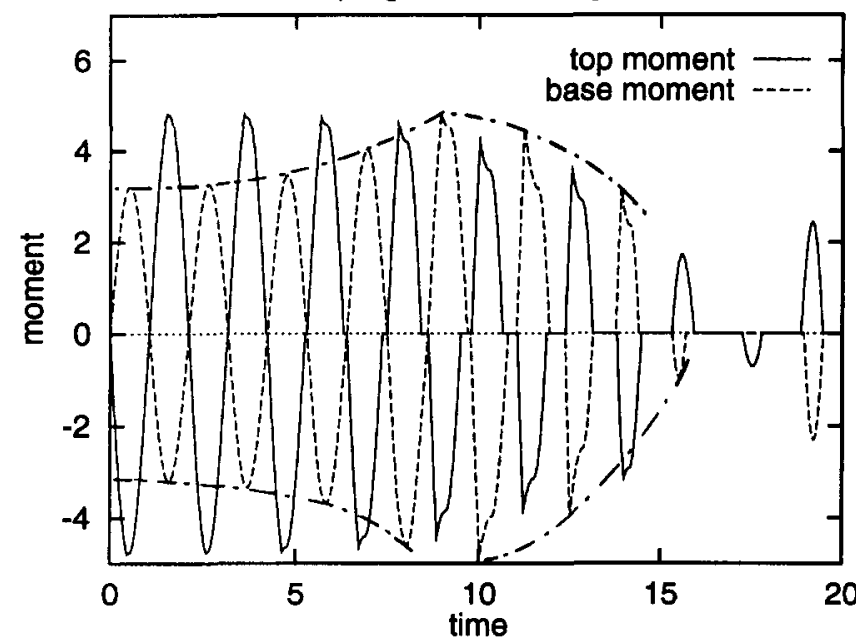

FIG. 7. Dynamic Solution of Column Subjected to Horizontal Sinusoidal Excitation: (a) Periodic (Elastic) Solution; (b) Solution with Progressive Damage

of each hinge remains the same, we have the exact general solution. Based on this solution, the response of the column to prescribed harmonic horizontal displacement history $q(t)$ at the column base is calculated. The initial conditions for each time interval are the displacements and velocities at the end of the previous time interval. The end of the current time interval is solved as the time at which the status of the hinge changes again, and the displacements and velocities calculated for that time are then used again as the initial values for the next interval. In this manner, the exact solution is easily constructed.

Fig. 7(a) shows the calculated exact histories of the bending moments at the base and at the top of the column for the case of elastic response. The column is excited by a prescribed sinusoidal history of the horizontal displacement at the base. This must lead to a periodic dynamic solution. The question is whether an instability in which the motion temporarily deviates exponentially from this periodic solution is possible. In contrast to the previous example of building floor, the periodic excitation does not lead to a symmetric dynamic solution with equal moment magnitudes at the top and bottom. To obtain a symmetric dynamic solution, symmetric excitations at both the base and the top would have to be prescribed. But this would be unrealistic. For this reason, the dynamically loaded column will seldom find itself near the static bifurcation state we just analyzed. However, the motion of the column approaches a

state corresponding to the limit point of the column statically loaded by the inertial forces, at which one of the hinges begins softening. In that case, temporary dynamic instability with exponential growth of deviations from a periodic solution must be expected.

In Fig. 7(a), the amplitude and period of the periodic excitation at base is selected so that the maximum moment exactly equals the peak moment $M_{p}$. Fig. 7(b) shows the solution under the assumption that the amplitude of excitation is slightly larger, which triggers softening response in the top hinge while the other hinge still behaves elastically. The peaks of the top moment cycles deviate progressively faster from the periodic solution and the deviation peaks grow approximately exponentially. This observation agrees with our previous general analysis of the initial dynamic response after the static limit point.

While the peaks of the top moment exponentially decline, the peaks of the base moment grow until the maximum bending moment is reached at the base (Fig. 7). Then the peaks of this bending moment start declining, too, and the deviation from the maximum moment grows approximately exponentially. This is not surprising because the start of softening at the base results in another negative eigenvalue of the tangential stiffness matrix. So this example clearly verifies the exponential growth of very small imperfections after softening has begun, and shows the growth can be quite rapid.

There is some similarity with the static bifurcation solution. First one hinge undergoes softening, then the other. In this case the solution would have a similar character even if the column were considered nonsymmetric.

\section{Localization in Sway of Portal Frame}

As another typical situation, consider that a large horizontal displacement $u$ is imposed in the portal frame in Fig. 8(a). Let $F$ be the corresponding horizontal load (or reaction). The frame has one statically indeterminate internal force. During plastic collapse, the frame becomes a single-degree-of-freedom mechanism with two plastic hinges, as shown in Fig. 8(b). However, when the diagram of the bending moment $M$ versus hinge rotation $\theta$ exhibits postpeak softening [Fig. 1(b)], the symmetric deformation mode with identical deflection curves of both columns (legs) and equal rotations of both hinges [Fig. 8(b)] is only one possible postpeak response to large imposed horizontal displacement. Another possible postpeak response is the deformation mode shown in Fig. 8(c), in which the softening damage localizes from two softening hinges into one hinge while the other hinge gradually unloads, behaving elastically.

For the sake of simple illustration, consider the momentrotation diagram to be linear, characterized by the peak bending moment $M_{p}$ and by rotation $\theta_{f}$ at which the bending moment is reduced to zero; see Fig. 1(b). By elastic analysis according to the principle of virtual work, the horizontal deflection and force at the limit of static elastic response (peak of the load-deflection diagram) are

$$
u_{p}=\frac{2+\lambda}{6} \frac{M_{p} H^{2}}{E I_{c}} ; \quad F_{p}=\frac{2 M_{P}}{H}
$$

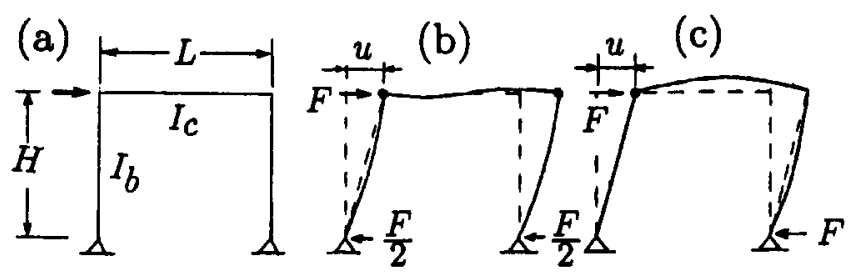

FIG. 8. Portal Frame, Softening Hinges, Symmetric and Nonsymmetric Deflections, and Load-Deflection Diagrams 
where $\lambda=L I_{c} / H I_{b}=$ a nondimensional parameter characterizing the ratio between the bending stiffness of the column and the beam; $H=$ height of the frame; $L=$ its span; and $I_{b}$, $I_{c}=$ centroidal moments of inertia of the cross sections of the beam and the columns (legs) in the frame. At the limit of elastic response, moment $M_{p}$ is reached simultaneously at both corners.

For the symmetric deformation mode with two softening hinges [Fig. 8(b)], the postpeak segment of the load-deflection diagram (Fig. 4) must be linear (because the moment-rotation diagram is linear) and must terminate with a state at which the rotations of both hinges are $\theta_{f}, F=0$, and the bending moments are zero everywhere. At that state, the displacement is $u_{f}=\theta_{f} H$.

For the nonsymmetric postpeak deformation mode, in which the left corner of the frame undergoes softening and the moment at the right corner decreases elastically from its peak value $M_{p}$, the terminal state (point 1 in Fig. 4) of the initial postpeak linear segment corresponds to the bending moment $M_{a}=0$ and hinge rotation $\theta_{a}=\theta_{f}$ at the left corner of the frame, while the rest of the frame is elastic. By elastic analysis according to the principle of virtual work, the deflection and the load at this terminal state (point 1 in Fig. 4) are

$$
u_{1}=\frac{2(1+\lambda)}{2+3 \lambda} H \theta_{f} ; \quad F_{1}=\frac{6}{2+3 \lambda} \frac{E I_{c}}{H^{2}} \theta_{f}
$$

At point 1, the horizontal reaction at the right support is equal to $F_{1}$ (because at the left support the reaction is zero). So the bending moment at the right corner of the frame is $M_{b}=F_{1} H$ $=6 E I_{c} \theta_{f} /[H(2+3 \lambda)]$, which must not be larger than $M_{p}$. From this we conclude that the nonsymmetric response path exists only if

$$
\theta_{f} \leq \frac{2+3 \lambda}{6} \frac{H M_{p}}{E I_{c}}
$$

Beyond point 1 , the frame behaves elastically as if there were a real hinge at the left corner. The load increases linearly up to point 2 (Fig. 4) for which elastic analysis according to the principle of virtual work gives

$$
u_{2}=\frac{1+\lambda}{3} \frac{M_{p} H^{2}}{E I_{c}} ; \quad F_{2}=\frac{M_{p}}{H}
$$

Then a softening hinge forms at the right corner and, as this hinge softens, the load decreases linearly to point $f$ in Fig. 4. The slope of the path from point 2 to point $f$ can be positive or negative (Fig. 4 shows the case of negative slope, representing snapback instability). The energy dissipated by each softening hinge up to total failure is $M_{p} \theta_{f} / 2$, and so the energy dissipated by the whole frame during failure is $W_{f}=M_{p} \theta_{f}$. This energy is also represented by the area under the complete loaddeflection diagram. Therefore, the areas $0 p f 0$ and $0 p 12 f 0$ in Fig. 4 must each equal $W_{f}$. So, the triangles $p 13 p$ and $32 f 3$ must have equal areas, which means that point 2 for nonsymmetric response must lie above the line $p f$ for the symmetric response.

The initial postpeak tangential stiffnesses of the frame, which represent the initial postpeak slopes, are obtained as $K$ $=\Delta F / \Delta u$ where $\Delta$ refers to the change from the peak state to point 1 or $f$. The stiffnesses for the symmetric and nonsymmetric responses are thus calculated as

$$
K^{(1)}=\frac{-F_{p}}{u_{f}-u_{p}} ; \quad K^{(2)}=\frac{F_{1}-F_{p}}{u_{1}-u_{p}}
$$

These stiffnesses can be either negative (as shown in Fig. 4) or positive. For a positive postpeak stiffness, there is snapback instability, which means the structure is unstable even under displacement control. For a negative postpeak stiffness, the structure is stable under displacement control.

As in the preceding problems, the actual postbifurcation path is that for which the compliance $1 / K$ is larger. So, the nonsymmetric path, labeled by superscript ${ }^{(2)}$, will occur if and only if

$$
\frac{1}{K^{(2)}}>\frac{1}{K^{(1)}}
$$

If we substitute here the foregoing expressions and rearrange them, we obtain the same inequality as (32), as might have been expected. Thus the hinge rotation at which the bending moment is reduced to zero [Fig. 2(b)] must be sufficiently small for the nonsymmetric response path to exist and to occur. It appears that condition (32) is often met in practice.

As might be expected, the dynamic response near the bifurcation state has a character similar to that calculated for the building floor. For identical sinusoidal excitations at the base of both columns, the static bifurcation state, with moments at both corners equal to the peak moment, can be approached by the dynamic solution.

The localization of softening damage into one hinge in the portal frame is, for example, relevant to the seismic analysis of bridge support bents. Because the same kinetic energy of earthquake is being absorbed by one instead of two plastic hinges, the dynamic collapse occurs faster.

\section{Localization in Multibay and Multistory Frames}

Earthquake damage in multibay frames with rows of identical columns often shows that only some of the columns in the row suffer serious damage. One reason for this phenomenon may again be dynamic localization of damage into fewer softening hinges. To illustrate this type of behavior, consider the one-story frame with infinitely many identical bays, as shown in Fig. 9(a). We analyze two basic simple cases:

1. Interior columns: For columns remote from the ends of the row, one may idealize the row of columns as infinite and assume the response to be periodic. When the peak moment $M_{p}$ is reached at the top of every column, the peak horizontal force per bay is $F_{p}=M_{p} / H$ and the horizontal displacement is

$$
u_{p}=\frac{M_{p} H^{2}}{E I_{c}} \frac{4+\lambda}{12}
$$

One possible mode of collapse is symmetric, with all the hinges softening simultaneously. For this mode, the final force $F=0$ and the final displacement $u_{f}=H \theta_{f}$. Another possible mode is that in which softening hinges alternate with unloading ones. It can be shown by elastic analysis that the moment in the softening hinges vanishes at

(a)
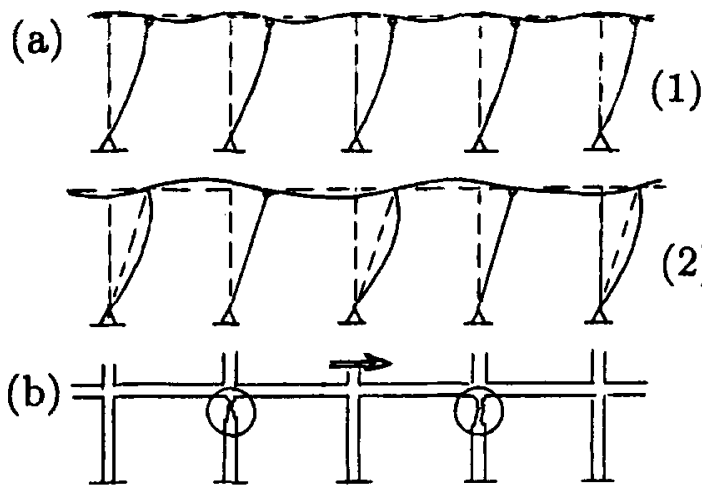

FIG. 9. (a) Symmetric and Nonsymmetric Deflections of Multibay Frame with Softening Hinges; (b) Damage Suffered 


$$
F_{1}=\frac{6}{4+3 \lambda} \frac{E I_{c}}{H^{2}} \theta_{f} ; \quad u_{1}=\frac{4+2 \lambda}{4+3 \lambda} H \theta_{f}
$$

This solution exists if the final moment $M_{1}=2 F_{1} H$ at the top of the unloading columns does not exceed $M_{p}$ so that these columns can really unload. Substituting $(37 a)$ for $F_{1}$, we can derive a localization condition

$$
\theta_{f} \leq \frac{4+3 \lambda}{12} \frac{H M_{p}}{E I_{c}}
$$

similar to (32). If $u_{1} \leq u_{p}$, there is snapback and the postpeak response is unstable even under displacement control. Comparing $(37 b)$ and (36) we conclude that the collapse mode with alternating hinges exhibits snapback for

$$
\theta_{f} \leq \frac{(4+3 \lambda)(4+\lambda)}{24(2+\lambda)} \frac{H M_{p}}{E I_{c}}
$$

2. Boundary columns: In real frames, even if the number of bays is very large, the moment distribution always differs from the ideally periodic one in the first and last few bays. Denoting by $M_{i}$ the moment at the top of column number $i$ in a semi-infinite frame, the values of moments in the "boundary range" derived by elastic analysis are given by

$$
M_{i}=\left(1-\frac{3 \lambda}{8+6 \lambda+4 \gamma} \gamma^{i-1}\right) F H
$$

where $i=1,2,3, \ldots ; \gamma=\sqrt{3+3 \lambda+9 \lambda^{2} / 16}-3 \lambda / 4-2$; and $F=$ average vertical force per column. As an example, the first five moments for a frame with $\lambda=1$ (equal stiffness of beams and columns) can be listed as follows:

$M_{1}=0.77353 F H ; \quad M_{2}=1.04263 F H ; \quad M_{3}=0.99197 F H ;$

$$
M_{4}=1.00151 F H ; \quad M_{5}=0.99972 F H
$$

Note that $-1<\gamma<0$ for any positive stiffness ratio $\lambda$, and so the maximum moment is always reached at the top of the second column. It might be expected that, in some situations, damage can localize into a single column, and the second column is the best candidate. Analysis of this assumed collapse mode indicates that snapback occurs if

$$
\theta_{f} \leq\left[\frac{1}{3}+\frac{\lambda(4+3 \lambda)}{28+24 \lambda+8 \gamma+6 \lambda \gamma}\right] \frac{H M_{p}}{E I_{c}}
$$

A stable solution with damage localized into one hinge can be expected for $\theta_{f}$ slightly larger than the right-hand side of the foregoing inequality.

The general conditions derived for an idealized frame with an infinite number of bays can be confirmed by numerical analysis of frames with a large but finite number of bays. As an example, consider a frame with 20 bays ( 21 columns), $L=$ $H$ and $I_{b}=I_{c}$, from which $\lambda=L I_{c} / H I_{b}=1$ and $\gamma=$ $\sqrt{3+3 \lambda+9 \lambda^{2} / 16}-3 \lambda / 4-2=-0.18826$. Let us state the results in terms of a nondimensional ductility parameter $\beta=$ $\theta_{f} E I_{c} / H M_{p}$. According to (38) and (39), a solution with alternating softening hinges is predicted for $0.486<\beta<0.583$, and according to (42), a solution with one softening hinge is predicted for $\beta$ slightly above 0.475 . Indeed, the numerical results show that: for $\beta \leq 0.4751$ snapback occurs after the simultaneous occurrence of the first two softening hinges in the second and penultimate column; for $0.4752 \leq \beta \leq 0.487$ damage localizes in the two most critical hinges under increasing floor displacement; for 0.488 $\leq \beta \leq 0.583$ damage localizes in every other hinge (starting from the second and penultimate one and progressing from both sides into the interior of the frame); for $0.584 \leq \beta \leq 0.589$ the final failure pattern shows 17 softening hinges; for $0.59 \leq \beta \leq 0.69$ the final failure pattern shows 19 softening hinges (all except the first and last one); and finally for $0.7 \leq \beta$ all the hinges undergo softening. We observe a very close agreement between the analytical prediction and the numerical results.

A similar localization of damage into fewer softening hinges can occur in multistory frames at any floor. The symmetric mode of collapse, in which hinges form simultaneously on the top and bottom of each column and rotate equally, is similar to elastic deformation and is exhibited in plastic (nonsoftening) response. If the hinges are softening, however, various collapse patterns with damage localized into fewer hinges are possible.

\section{COMMENTS ON SIMPLIFIED EARTHQUAKE ANALYSIS}

Aside from the need to check the singularity of the tangential stiffness matrix and analyze a possible breakdown of symmetry of dynamic response in finite-element programs, we should also realize the consequences of the present results for practical simplified analysis of earthquake response (Chopra 1995). Such analysis is normally based on approximating the entire structure by an equivalent single-degree-of-freedom oscillator whose stiffness and mass are chosen so as to approximate the first vibration mode of the structure taking into account the stiffness reduction due to damage ("Seismic" 1986).

In light of the present results, however, such an approach can be applicable only for moderate damage occurring when the softening hinges begin to form. It cannot be applicable to the analysis of complete collapse, because the exponential growth of the deviation from the symmetric mode of response represents a mode that is very different from the initial response mode approximated by the single-degree-of-freedom oscillator.

Perhaps a simplified method based on an oscillator with two degrees of freedom, one representing an equivalent system for the initial first mode of vibration and the second representing an equivalent system for the deviation from the symmetric response after the static bifurcation state, could be developed.

\section{CONCLUSIONS}

1. Under monotonic static loading, softening damage in frame structures causes bifurcations of the equilibrium path in which the symmetry of response breaks down and the damage localizes into fewer hinges which soften faster. Although the dynamic response of a structure with softening damage (and with fixed parameters) cannot exhibit bifurcation in time, it becomes (for a limited time, temporarily) dynamically unstable (in the sense of Liapunov) after the static bifurcation state has been passed. Small imperfections cause deviations from the primary (symmetric) response mode to grow exponentially (at least temporarily), causing dynamic localization of damage into fewer softening hinges. Because the same kinetic energy must be absorbed by fewer inelastic hinges, the collapse then progresses faster.

2. An exponentially growing deviation from a periodic solution, causing a similar behavior, also occurs in the dynamic response near the static limit point. (The bifurcation point may, but need not, coincide with the limit point.)

3. The softening-induced (time-limited) dynamic instability due to formation of softening hinges may have serious implications for the seismic resistance of building frames and bridges. The inelastic hinges in reinforced-concrete columns or prestressed beams exhibit postpeak softening, caused by compression failure of concrete. Steel beams can also exhibit postpeak softening, caused by elastoplastic buckling of flanges and webs or by growth of 
cracks during earthquake. Such behavior generally leads to the aforementioned type of damage localization.

4. Localizations of damage into fewer softening hinges which lead to exponentially growing deviation from a symmetric or periodic solution have been demonstrated for the following typical examples: torsional rotation of a building floor, horizontal shear of a building column, shear loading of a portal frame, shear loading of a multibay frame, and shear loading of a multibay-multistory frame.

5. Dynamic localizations due to softening hinges cannot be captured by analyzing the structure as a single-degreeof-freedom oscillator, because the deviation from symmetric response represents a very different mode of response.

\section{ACKNOWLEDGMENTS}

Partial financial support under U.S. National Science Foundation Grant MSS-911-4476 to Northwestern University is gratefully acknowledged. Further support for material models of softening cracking damage in concrete has been received from the Center for Advanced Cement-Based Materials at Northwestern University. Milan Jirásek has been supported by Grant No. 1311320 from the Czech Technical University.

\section{APPENDIX. REFERENCES}

Bažant, Z. P. (1976). "Instability, ductility and size effect in strain-softening concrete." J. Engrg. Mech., ASCE, 102(2), 331-44.

Bažant, Z. P. (1988). "Stable states and paths of structures with plasticity or damage." J. Engrg. Mech., ASCE, 114(12), 2013-2034.

Bažant, Z. P., and Cedolin, L. (1991). Stability of structures: elastic, inelastic, fracture and damage theories. Oxford University Press, New York, N.Y.

Bažant, Z. P., and Cedolin, L. (1993). "Why direct tension specimens break flexing to the side." J. Struct. Engrg., ASCE, 119(4), 11011113.

Bažant, Z. P., and Kazemi, M. T. (1994). "Localization of softening damage in frames and implications for earthquake resistance." Proc., 5th U.S. Nat. Conf. on Earthquake Engrg., Earthquake Engrg. Res. Inst., Oakland, Calif., Vol. 1, 313-322.

Bažant, Z. P., Pan, J.-Y., and Pijaudier-Cabot, G. (1987a). "Softening in reinforced concrete beams and frames." J. Struct. Engrg., ASCE, $113(12), 2333-2347$.
Bažant, Z. P., Pijaudier-Cabot, G., and Pan, J.-Y. (1987b). "Ductility, snapback, size effect and redistribution in softening beams and frames." J. Struct. Engrg., ASCE, 113(12), 2348-2364.

Borre, G., and Maier, G. (1989). "On linear versus nonlinear flow rules in strain localization analysis." Meccanica, Italy, 24(1), 36-41.

Chopra, A. K. (1995). Dynamics of structures: theory and applications to earthquake engineering. Prentice-Hall, Inc., Englewood Cliffs, N.J.

de Borst, R. (1989). "Numerical methods for bifurcation analysis in geomechanics." Appl. Mech. (Ingenieur-Archiv), Berlin, Germany, 59, 160-74.

Delallera, J. C., and Chopra, A. K. (1994). "Accidental torsion in buildings due to base rotational excitation." Earthquake Engrg. and Struct. Dynamics, 23(9), 1003-1021.

Delallera, J. C., and Chopra, A. K. (1995). "Understanding the inelastic seismic behavior of asymmetric plan buildings." Earthquake Engrg. and Struct. Dynamics, 24(4), 549-572.

Guckenheimer, J., and Holmes, P. (1983). Nonlinear oscillations, dynamical systems, and bifuncations of vector fields. Springer-Verlag, Berlin, Germany.

Hunt, G. W., and Baker, G. (1995). "Principles of localization in the fracture of quasi-brittle structure." J. Mech. and Phys. of Solids, 43(7), $1127-1150$.

Leroy, Y., and Ortiz, M. (1989). "Finite element analysis of strain localization in frictional materials." Int. J. Numer. and Anal. Methods in Geomech., 13, 53-74.

Maier, G. (1971). "Instability due to strain softening." Stability of Continuous Systems, Proc., IUTAM Symp., Springer-Verlag, Berlin, Germany, 411-417.

Maier, G., and Zavelani, A. (1970). "Sul comportamento di aste metalliche compresse eccentricamente. Indagine sperimentale e considerazioni teoriche," Costruzioni Metalliche, 22(4), 282-297.

Maier, G., Zavelani, A., and Dotreppe, J. C. (1973). "Equilibrium branching due to flexural softening." J. Engrg. Mech., ASCE, 99(4), 897 901.

Rots, J. G., and de Borst, R. (1989). "Analysis of concrete fracture in direct tension." Int. J. Solids and Struct., 25(12), 1381-1394.

Runesson, K., Larsson, R., and Sture, S. (1989). "Characteristics and computational procedure in softening plasticity." J. Engrg. Mech., ASCE, 115(8), 1628-46.

"Seismic design guidelines for essential buildings." (1986). Rep. DATM-5-809-10-1, Dept. of Army, Navy and Air Force, Washington, D.C.

Stolz, C. (1989). "On some aspect of stability and bifurcation in fracture and damage mechanics." Cracking and damage: strain localization and size effect, J. Mazars and Z. P. Bažant, eds., Elsevier, London, England, 207-216.

Wiggins, S. (1990). Introduction to applied nonlinear dynamical systems and chaos. Springer-Verlag, New York, N.Y. 